were removed. The glands were enlarged, but did not exhibit the characters of cancer, either to the naked eye or to the microscope.

Thus far, with the exception of Case Iv, from whom I removed a second epithelioma on the stump of the tongue, all the patients remain well and free from recurrence of the disease-a striking testimony to the advantage of very early operation for the removal of epithelioma of the tongue. The glands were removed in 5 of the 7 cases, the exceptions being Case o and Case III. I should like to have removed them in every instance, for I have lost a patient within the last few months from secondary affection of the glands, which did not make its appearance for more than three years after the successful removal of one of the smallest cancers of the border of the tongue that I have ever seen.

Let me recapitulate the forms under which the disease commenced in these 7 cases. They are four in number:

1. A flat, very slightly raised. smooth, red glazed plaque, feeling like a thin piece of gristle in the surface of the tongue, not thicker than a threepenny or sixpenny piece, and looking and feeling just like a primary hard sore. (Cases $o$ and 1I, to which may be added Case IV as a probable further stage of the same condition.)

2. A white, warty growth, not ulcerated, and scarcely indurated at its base. (Case I.)

3. A slight thickening and hardening of an old leucoplakic area, rather distinguishable to feel than sight, very likely an earlier stage of No. 1. (Case III.)

4. A nodular plaque, red, and commencing to ulcerate, with drawing-in of the surrounding tisses. (Case vi.)

Two years ago, before my eyes were opened by the work of the Imperial Cancer Research, I should have regarded five out of the seven as precancerous conditions, and I am now wondering whether there are really any conditions perceptible to human sight and feel which are precancerous in the sense in which I have been accustomed to employ the term.

Many persons will wonder why I have been so slow to discover the true nature of these conditions. I can only answer that I am humiliated at my ignorance, and can only plead, in excuse for it, that I could scarcely ever, a few years ago, persuade patients to submit to operation for these conditions. And, if I had removed them, it is quite possible that their real nature might have been overlooked unless they had been examined in continuous sections after the manner which is now commonly practised in the investigation of cancerous and doubtful growths.

\section{(Clinital hamarks}

\section{EARLY RECOGNITION OF CANCER OF THE BREAST.}

By G. LENTHAL CHEATLE, C.B., F.R.C.S.,

SURGEON TO KING'S COLLEGE HOSPITAL, ITALIAN HOSPITAL, AND THE HOSPITAL FOR EPILEYSY AND PARALYSIS, MAIDA VALF.

There are cases of cancer of the breast in which the tumours are so small that a positive diagnosis of cancer is rendered doubtful by unaided clinical signs. The management of these cases requires earnest reconsideration. The importance of the matter is increased by the knowledge that a small cancer need not necessarily be an early one and if one considers upon what the clinical signs of rancer of the breast depend, one must at once realize that the definite and classical clinical signs-even the earliest of them-owe their existence to a degree of spread of the disease which must reach certain stages before the signs for which we so anxiously search are recognizable.

In hospital and private practice the majority of the breast cancer cases come for consultation and operation after they have been watched for periods that vary from about two to twelve months, and often much longer. It is needless to point out how invaluable is the time lost in these months, for during the watching period cases must frequently pass from the curable into the incurable stages, and in the early treatment of cancer the saving of time means the saving of life.

Therefore, the ideal management of a case in which the clinical signs of cancer are not clear is not to wait until the cancer has spread sufficiently to render them so, but to settle the matter at once by a complete macroscopical and, if necessary, microscopical examination, the essential details of which I shall allude to later. To illustrate the point of view which needs expulsion from our methods I will relate the following type of case: A woman at the age of 27 years consulted a surgeon (with her medical man) three years ago with regard to a small tumour in her breast; the surgeon told her it might or might not be cancer, and for the present he "would give her the benefit of the doubt" and would watch her case. A year after, when the clinical signs of cancer had become undoubted, the breast and its lymphatic vascular system were as thoroughly removed as possible, but two years after the operation she died with cancer in the lungs, liver, and a rib; there was no evidence of disease in the area of the operation. Now, it is quite obvious that by adopting the waiting and watching policy the surgeon responsible for the management of this case did not give his patient "the benefit of the doubt." "The benefit of the doubt" would have been given her by clearing it up, which at that time could have been done only by a more complete examination. The diagnosis of cancer would have been made. The complete operation would then have been performed. One year of the cancer's spread would have been stopped, and possibly her life would have been saved.

It must be understood that on the conclusion arrived at from the evidence obtained by the first examination will depend the future management and probably the future safety of the case.

In this article I propose to demonstrate :

1. How to examine a breast case clinically, and at the same time point out how the examination must lead to the certainty of malignant disease in some cases and to uncertainty of it in others, especially those in which the cancer is too small to give rise to the typical signs of its presence-that is, the very cases which it is so essential to diagnose and treat early.

2. The management of a case in which theclinical examination leaves the positive diagnosis of a small cancer uncertain.

\section{The Examination.}

The best position for the patient is flat on the back and on a fairly high table. I regard the recumbent posture as a great aid in the diagnosis of all diseases of the breast. Both breasts must be well exposed for the comparison of the abnormal with the normal side.

\section{A.-Palpation of the Breast.}

The breast tissue must not be picked up, but must be palpated by the soft pads on the palmar surfaces at the top of the fingers, by which both breasts should be gently rolled over the underlying thorax. Any swelling, nodule, or irregularity which is discoverable clinically will be at once felt. The object of this examination is to discover:

1. Whether both breasts cantain nodules, swellings, or irregularities.

2. Whether the breast complained of be the only one affected, and if so, whether there is one or more than one lesion in it.

The observations have the following value in building up a diagnosis for or against cancer.

Should multiple nodules or swellings or irregularities be found in both breasts the diagnosis is probably one of chronic mastitis or cystic changes, or combined cystic changes and chronic mastitis.

Should multiple nodules or swellings or irregularities be found in one breast only, the diagnosis is, with some exceptions to which I shall refer later, in favour of chronic mastitis or cystic changes, and not cancer. But should only one nodule or swelling or irregularity, or even a swelling surrounded by a small area of irregularity, be found in one breast, it is probably a tumour, and hence may or may not be cancer.

The same conclusion applies to one nodule, swelling or irregularity in each breast; both breasts being affected is a sign per se, which is rather more in favour of chronic mastitis than cancer. But although cancer in both breasts is rare, one of the breasts may contain cancer, hence too much mental bias must not be allowed in any direction when one lesion exists in each breast.

\section{B.-Retraction of the Skin.}

Having discovered swellings or irregularities in both breasts or only in one breast, or having discovered one swelling, nodule, or irregularity in one breast only, the 
next point to be decided: is: Can the skin covering the abnormal part or parts be demonstrated to retract? The sign is best elicited by gradually and gently pushing the breast towards that part of the skin to be tested. The test is most delicate when the tumour is not itself pushed but when it is pushed indirectly by the fingers applied to a part of the breast well away from the seat of the suspected area. If present, the slightest retraction of the skin covering the suspected swelling can be noted and must be caused.by the pull on it of the underlying tumour, since the wrinkle of the skin at that spot could not be caused by direct pressure of the tingers, because they would be too far away to induce it.

Retraction of the skin over a tumour, induced with these precautions, is one of the earliest and most constant of the classical clinical signs of cancer. The same rule applies when the covering skin retracts over multiple nodules swellings, or irregularities which have been discovered in one breast only. In the latter case it is probably an advanced case of diffused cancer of the breast, in which the enlarged glands, if present, cannot be felt. Fig. I is from a case in which cancer was diffused throughout the gland. Only one breast was affected with multiple nodules and irregularities; no enlarged lymphatic glands could be felt, and the correct diagnosis of cancer was made solely on the retracted skin and flattening of the normal curves of the breast.

As yet I have never seen multiple nodules, swellings, or irregularities in both breasts accompanied by retraction of the covering skin. I have seen two cases in which one tumour occurred in each breast, in both of which the covering skin was adherent and both tumours were cancer, but the condition is rare. Retraction of the skin is caused in three ways: First, by the invasion by the cancer of the fibrous bands (ligamenta suspensoria of Sir Astley Cooper), which pass from the breast capsule to the skin (see Fig. I); secondly and later, by the adherence of the tumour itself to the skin (see Fig. II); thirdly, it may also be caused by the attachment of the tumour to the underlying pectoral fascia, in which case any attempt made to move the breast or pectoralis major will induce retrac-

tion of the skin by the pull on it of the normal fibrous connexions between it and the capsule of the breast.

The first is the most commonly seen, and the third the rarest.

\section{C.-Flattening of Part or the Whole of a Normal Curve of} the Breast.

This sign, which is partly a variation of the test just described, is due mainly to the same causes, and when present it is also a very delicate and trustworthy test of cancer. I have never seen it alluded to. The flattening of a curve can be seen best when the particular curve is viewed from any position which is on a level with it, hence the surgeon must lower his head to bring his eyes to a level with the breast, and upon so doing the slightest loss in the curve is then seen with marked accuracy. (See Figs. I and II.)

It may here be stated that when these three signs are associated the case ought to be regarded as one of cancer even in the absence of all other signs. The facts are so important it may be .well to repeat them :

1. A single tumour in one breast only (the opposite breast normal).

2. Retraction of skin over the tumour.

3. Flattening of part or whole of the curve over the tumour.
It must not, however, be assumed that difficulties in diagnosis never occur. In somewhat rare cases tuberculosis, syphilis, actinomycosis, slowly-acting microorganisms of suppuration, especially when they attack a thick-walled galactocele, all may occasionally simulate the clinical signs of cancer. In my experience the one or two cases of suppurating galactoceles which resembled cancer occurred close up to the nipples.

\section{D.-Examination of Iymphatic Glands in the Axillary and Supraclavicular Region.}

I take it that, however short the duration of the cancer, as soon as the axilla (or supraclavicular) glands are felt enlarged, hard, and discrete, the disease is in an advanced state and the clinical diagnosis a matter of ease and the prognosis a matter of extreme doubt. The method of diagnosis in advanced cancer of the breast is not the object of this article, therefore I leave the sign with a hint to examine both axillae always, for if BOTH sides contain enlarged lymphatic glands it would be a point against the diagnosis of a "beginning" cancer.

\section{E.-Retraction of the Nipple and Haemorrhage from the \\ Nipple.}

Retraction of the nipple as a sign of cancer of the breast is too much sought for, too long waited for, and when found it; value is overestimated. It occurs in cancer early when the disease begins under or near the nipple, otherwise it is a comparatively late sign, and its absence does not materially affect a diagnosis. The value of a retracted nipple, when present, in a case for diagnosis is lessened by the knowledge that it may always have been so in one or both breasts; and although the history of the case may prove a nipple has been retracted only recently (and as such is of some value), it must be remembered that chronic mastitis, cysts, and other tumours near the nipple can cause its retraction. The fibro-adenoma in Fig. III caused a rapidly-increasing retraction of the npiple.

Haemorrhage from the nipple when it is gently squeezed must be regarded as a serious sign when no inflammatory nor obvious cause is present to account for it, and in these circumstances the cause should be at once determined. I cannot say that I recognize what the so-called "duct cancer" is, but I am perfectly familiar with the tumour structure to which some observers are inclined to limit the term, and I should have expected this tumour to induce haemorrhage from the nipple, at least occasionally. But I have seen these tumours structurally pure and also mixed with the so-called alveolar or scirrhus variety, and as yet I have never seen one cause haemorrhage from the nipple. I have, indeed, seen a case of cancer of the breast in which there was haemorrhage from the nipple; but the cancer was of the pure alveolar or scirrhus type, which was complicating Paget's disease of the nipple in the same breast. Clinically it is not possible to diagnose the so-called "duct cancer" tumour from the alveolar or scirrhus types or from a tumour composed of a mixture of the two types. At one time I began to think one could, but now I am quite sure it is impossible. Some observers say that "duct cancer" is less malignant than the other type; but Fig. I, which is a pure so-called "duct cancer," was a highly malignant tumour, and another case which contained a mixture of the two types, was one of the most fulminating malignant tumours of the breast I have ever seen or heard of. Again, looked at as naked-eye preparations, Figs. IV and $\mathrm{V}$ look like tumours composed structurally of the same tissue ; but 


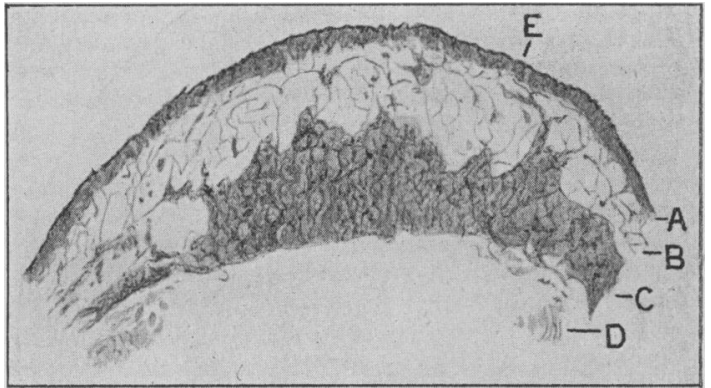

Fig. 1.

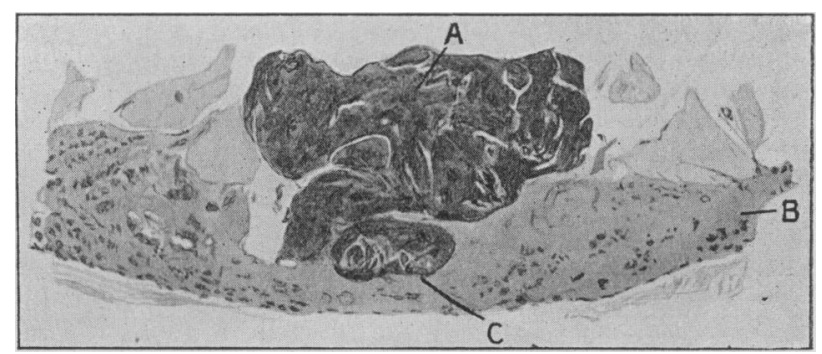

Fig. III.

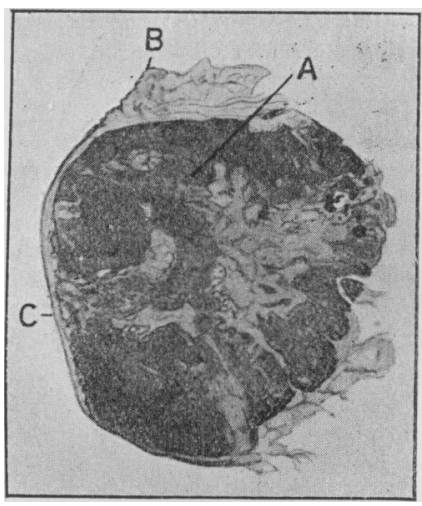

Fig. II.

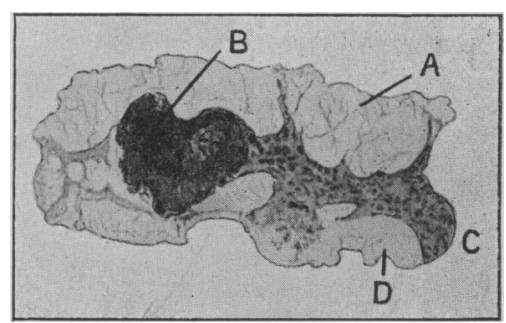

Fiø. VIII.

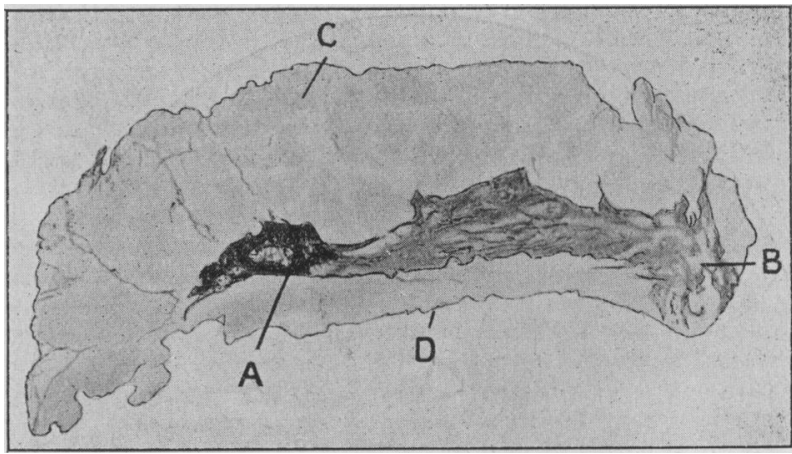

Fig. VI.

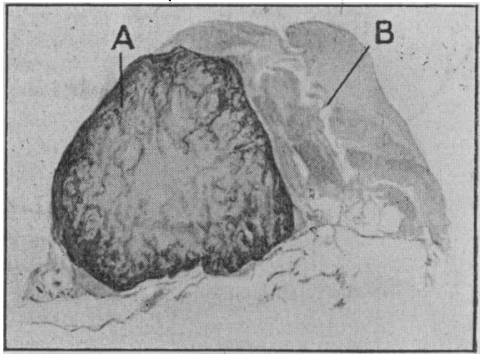

Fig. IV.

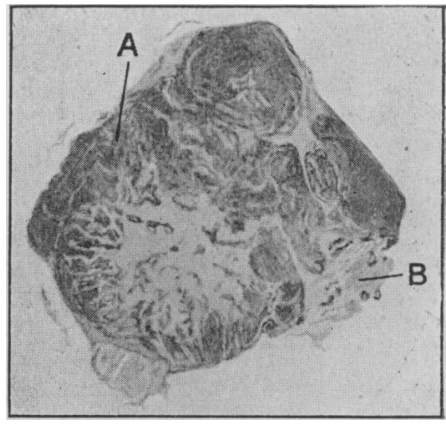

Fig. V.

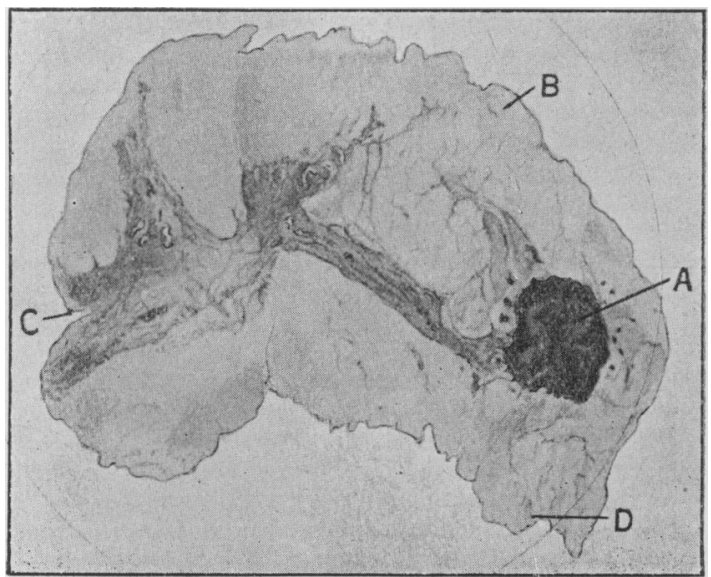

Fig. VII.

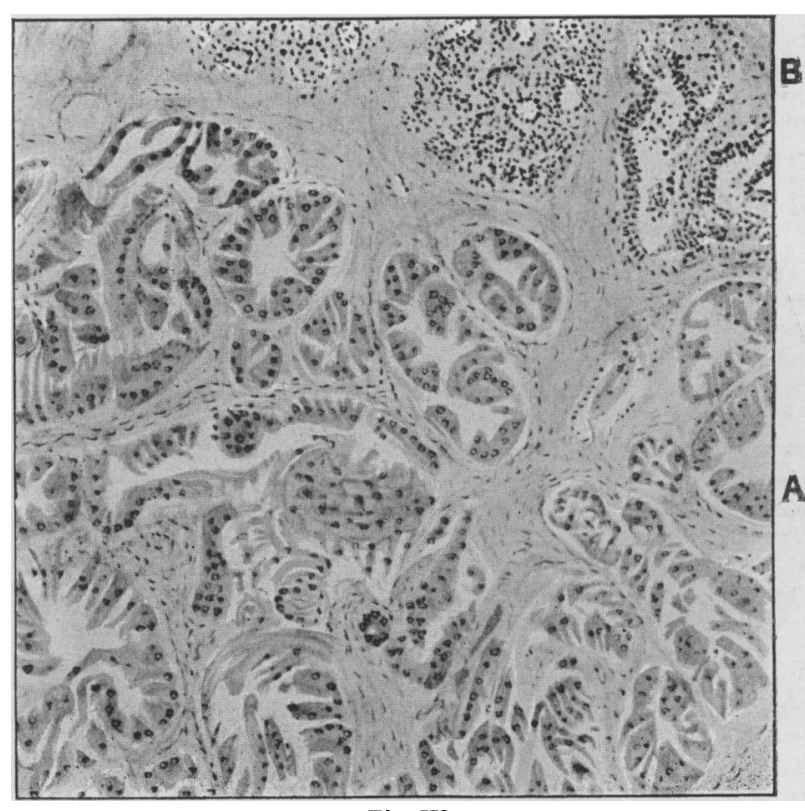

Fig. IX. 


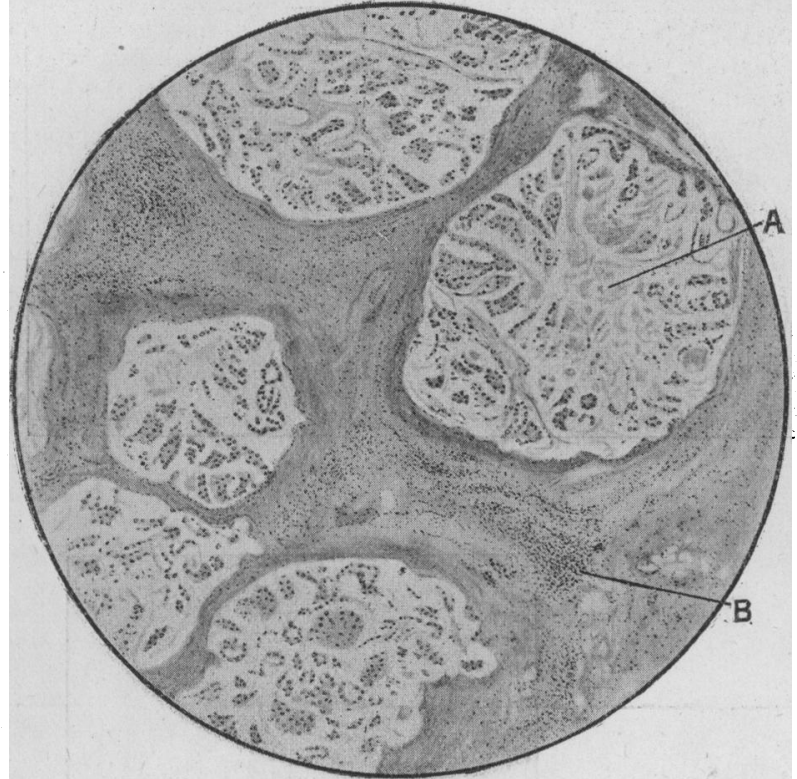

Fig. $\mathbf{x}$

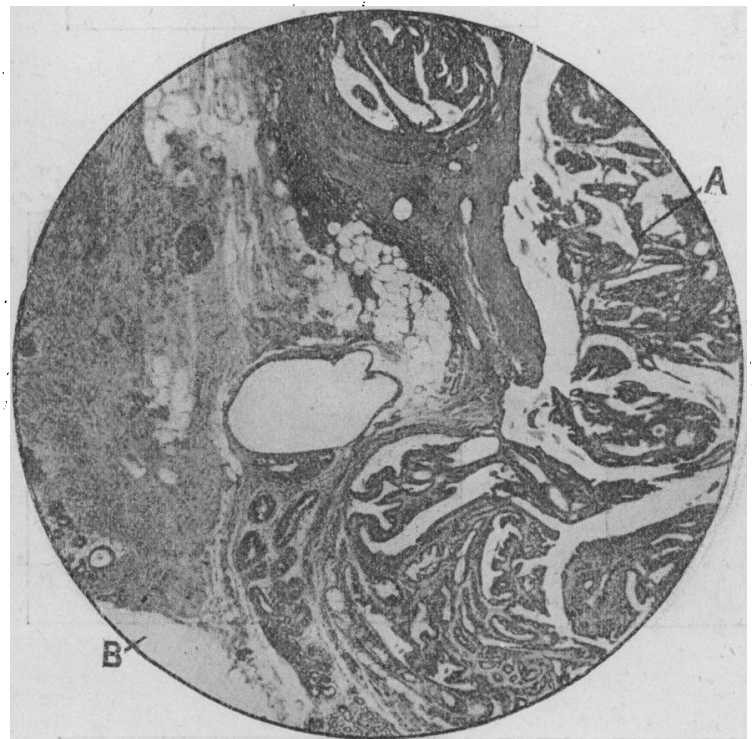

, Fig XI

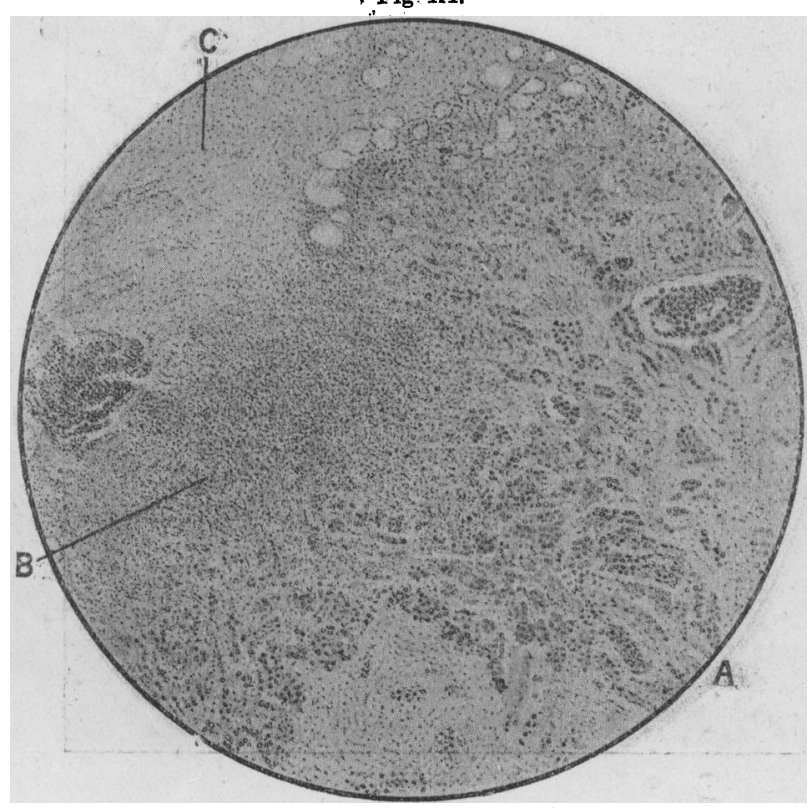

Fig. XII.

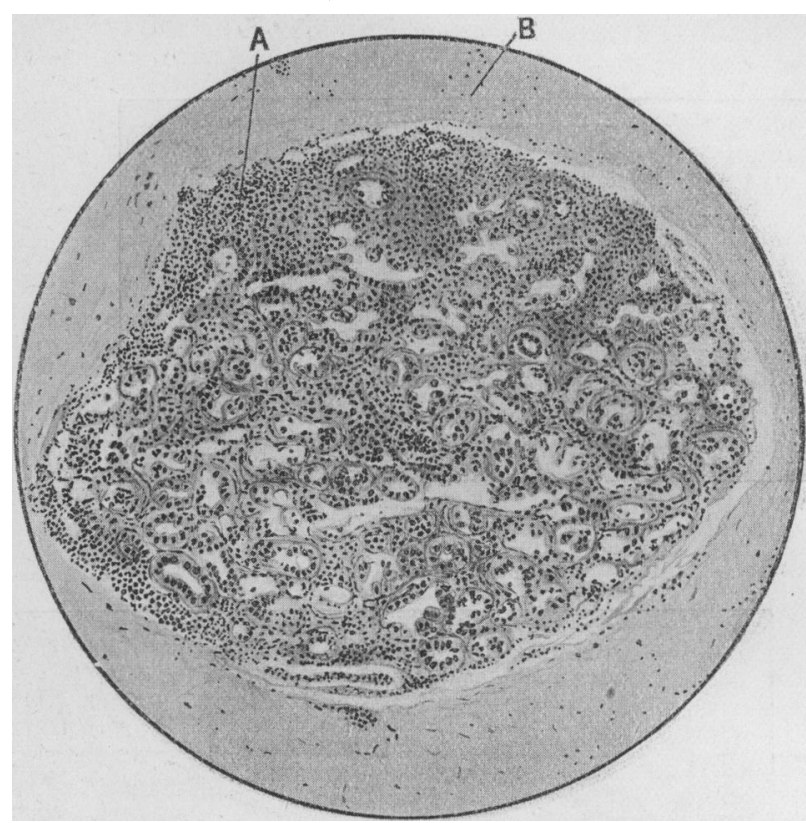

Fig. XIII.

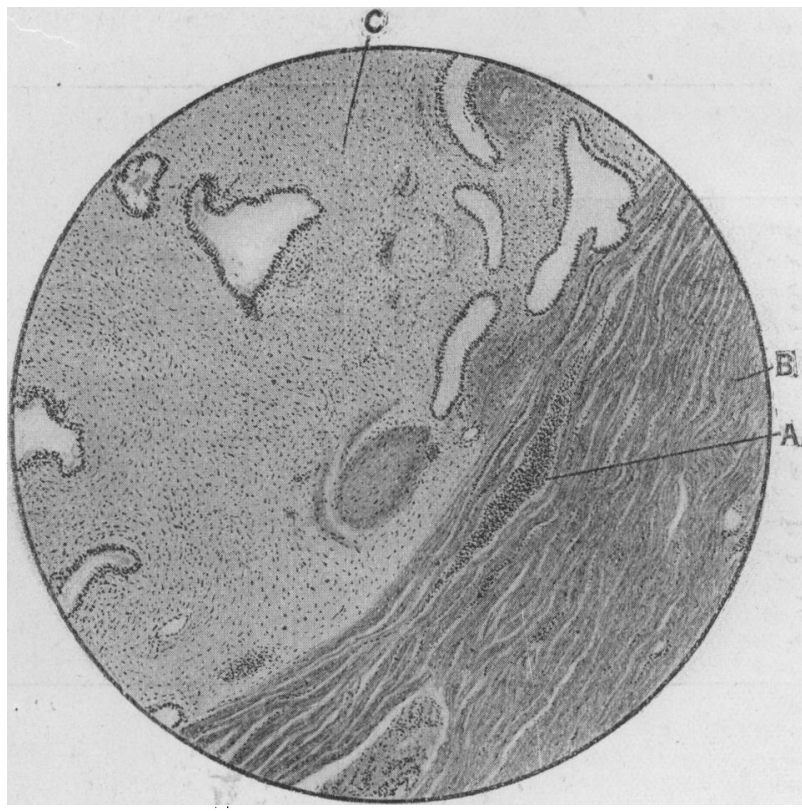

Fig. XIV

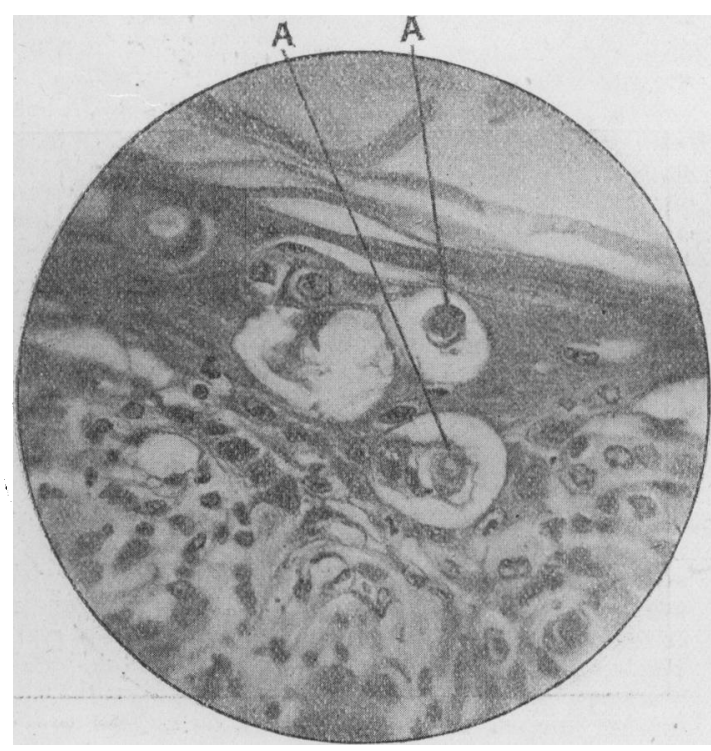

Fig. XV 
Fig. IV is an alveolar or scirrhus type and Fig. $V$ is the so-called "duct cancer" type. They were in the wards at the same time; clinically they almost exactly resembled one another, and until I had the two growths under the microscope I thought they were probably identical in structure.

The question of duct cancer will be again referred to ; but now, having discussed the comparative values of the main guides to the early recognition of cancer of the breast in which the classical clinical signs are as nearly conclusive as any clinical signs can be, I propose to demonstrate what are the signs that ought to make one doubt as to whether the case is or is not one of cancer.

The main and important points clinically observable in such a case are these: There is a small, possibly tender, swelling, and its immediate neighbourhood may be sur rounded by some irregularity in one breast only, or there may exist in only one breast one possibly painful or tender area of irregularity. There is no retraction' of the skin covering the suspicious area, nor is there discernible any flattened interruption of the curve over it, and the rest of the same breast and the opposite breast are normal on palpation, and all other clinical signs are absent. No matter what is the duration of these signs this is the clinical picture of the doubtful case. All one can say about it clinically is that being so isolated it probably is a tumour, but whether it is malignant or not there are not sufficient data to say; nor can one be quite sure it is not, after all, merely an inflammatory affair, and all that can be said on that point is, it may be inflammatory, but being so isolated a lesion one does not think so. It may, however, be said that recent trauma may cause the actual signs I have indicated; but were it so there would be a bruise on the skin; and the imagined or real history of injury is so common in cases of cancer of the breast that it cannot be trusted to negative the diagnosis of cancer unless supported by other clear and detectable signs.

With regard to the management of a doubtiul case I quietly and gently put the matter before the patient and advise that I should be allowed to clear up the issue by further examining the swelling or irregularity, as the case may be. My advice has always been taken, and I must say that in my experience the early-detected cases of cancer are more numerous than the cases that turned out to be merely of a nonmalignant nature - the cases of cancer decidedly berefited by giving the true "benefit of the doubt." And the cases in which the disease was found to be not malignant were at once relieved of a terrible mental anxiety.

Now by a further examination I mean the removal of the suspected tissue for direct macroscopical and, if necessary, microscopical evidence. If the positive diagnosis of cancer can be made upon the macroscopical appearances, and in many cases of cancer this can be done, the operation of total removal of the breast can be performed at once. But the difficulty of the macroscopical evidence sometimes lies in the inability to say from it that the lesion is not a cancer, hence, under these circumstances a complete microscopical examination must be made before any further operation is attempted.

The method of removal of the tissue in doubt is one of great importance, and unless done with due precautions against disseminating the growth by transplantation it should not be done at all. It must be remembered that the condition may be cancer, and by cutting into the swelling and excising a piece of it, or by cutting the swelling out by an incision carried immediately around it, one will be courting disaster. A limited operation of this kind, conducted under local anaesthesia or gas, or even as a preliminary measure to the complete operation for the removal of the breast, is a dangerous proceeding.
My method is this: As a rule an elliptical-shaped piece of skin is mapped out, measuring 3 in. at its widest point, which is placed immediately over the suspected area. This elliptical-shaped piece of skin is left untouched, but the skin around it is undercut in all directions, and that half of the breast which contains the suspected area is excised, together with the underlying part of the fascia covering the pectoralis major, and the elliptical piece of skin comes away undisturbed, with the parts thus removed. The resulting cavity is then soaked with a 1 in 500 watery solution of perchloride of mercury, the wound is sewn up and temporarily covered over with a layer of gauze during the macroscopical examination of the suspected area, and if cancer is evident I change my gloves and instruments, and proceed at once to remove the breast.

The intention of these methods is easy to understand. It is this: The suspected area may contain cancer, and, if so, the disease will be spreading towards the skit above and the fascia beneath and in the breast around; therefore, to avoid the possibility of disseminating the growth by transplantation, the piece to be examined is thus comparatively extensively removed with the parts containing it. The final soaking of the tissues with the perchloride solution is done with the object of killing any cells of cancer that may have been liberated.

I need not trouble the reader with the technique of preparation should a microscopical examination prove necessary, but the tissues are carefully embedded in paraffin, and whole sections of them are cut and a most methodical and deliberate examination is carried out. I am a disbeliever in the microscopical examination on the "while-you-wait" system at the time of the operation as advised and practised by some surgeons. As the operation for removal of the doubtful area is undertaken for the sake of accuracy, there is every reason to avoid a hurried examination of an inefficiently stained section from which important cells may have fallen during its hasty preparation.

Figs. VI, VII, VIII are cancer-containing sections of parts removed from three breasts which clinically gave the doubtful indications to which I have above referred in detail, and curiously enough the cancer in all three cases is situated at the breast's margin. The case from which Fig. Vl was taken had, as the only clinical signs, a slightly tender 'irregularity, and there were no changes in the skin or curves of the breast over it, and no other abnormal condition could be felt in the same breast or in the opposite breast, and was one of those cases in which macroscopically the diagnosis could not be made. In the cases from which Figs. VII and VIII were taken, a single tender swelling could be felt, but no change could be seen in the skin and curve over them, but after their - removal as above described the macroscopical evidence was enough to definitely prove cancer. The cancer seen in Fig. VIII was lying under the edge of the pectoralis major almost in the axilla, a most difficult and obscure place to detect early cancer in a fat subject.

I propose now to point out several pathological conditions which one discovers in the parts removed for microscopical diagnosis, and from them it will be seen that it is not always easy to make at once a dogmatic statement upon a section, even if it has not been prepared hurriedly, and that more than one section and more than one stain may be necessary for the purpose.

1. A common lesion is seen in Fig. IX. It is, I believe, an early cystic change, which, at this stage, is marked by much epithelial activity ; in some of the small alveoli, which are crammed" with epithelial cells-such an alveolus is seen in Fig. IX-there are cells undergoing a 
somatic mitosis. Although in parts the appearance suggests an early stage of cancer, I am sure it is not; but I believe this benign pathological change in the alveoli, which may become filled with papillomata, may form one of the sources of origin of the so-called " duct cancer." Fig. X (from Fig. I) is a tumour which showed the same type of tissue, but in its malignant form. However, the benign early cystic change which I am now describing is quite a common lesion which exists most typically as the only pathological change in the breast; but it can also be seen in the breast immediately surrounding some fibroadenomata, and such a condition exists at one part of the margin around the fibro-adenoma in Fig. III. I have also discovered it in breasts removed post mortem from women whose ages ranged from 30 to 50 , and who before death never, so far as known, complained of any breast trouble. If anybody seriously advocates removal of all breasts in which this lesion exists, on the ground that it contains epithelial cells in a state of marked activity, and because, as I believe, it may become one of the sources of origin of the so-called "duct cancer," then all women from 30 years onwards ought to subject themselves to this treatment, for in most cases, except from microscopical examination, the lesion would not be discoverable. The idea is thus rendered preposterous.

2. Another common pathological change seen in the suspected areas : of breasts removed from patients aged from about 30 to 50 years is that seen in Fig. XI. It is generally a microscopic lesion, but in one of my cases it was as big as half a split pea, and caused a sufficient irregularity to be detected clinically, and was the seat of slight tenderness. I have seen the same condition in breasts removed post mortem, in chronic mastitis, and in some of the breasts just described in the paragraph above and also in parts of the breast tissue immediately around some fibro-adenomata.

I regard this condition as a simple and benign condition, due to a papillomatous change in the ducts of the breast, which can be accurately traced in various stages of development. Although it is not malignant, I can quite understand that the so-called "duct cancer" can and probably does originate from this structure, as well as in the papillomat ous cystic change which begins in the alveoli which I have just described. However, upon being asked whether this lesion can be called a "precancerous condition" with the object of insisting upon early and preventive treatment, I should again say it is absolutely impracticable, for the following reasons: In the great majority of cases in which $I$ have seen it the lesions have been microscopical and found by accident, and so small and harmless that they were clinically undiscoverable, and as such one occasionally comes across them in post-mortem breasts of women who complained of no mammary trouble.

Lastly, when the breast tissue of those cases which contain cancer is carefully examined, it is very common to find in it no trace of this lesion or that described in paragraph 1 . On the other hand, I must admit in some cases the cancerous breast does contain the lesions described in paragraphs 1 and 2 , but it is by no means the rule. The most practicable method of dealing with them is to remember that directly they attain sufficient size to be recognized as a swelling or an irregularity, upon a clinical examination, they will give rise to doubt as to whether they are or are not obscure cases of cancer and the doubt can be at once cleared, and the lesion appropriately dealt with by a further examination conducted on the lines I have definitely indicated.

3 . The presence of lymphocytes and plasma cells around the margin of the part removed for microscopical diagnosis is of value in favour of the part being cancer only when they are seen in overwhelming numbers and crowding into and among the cells of the lesion. (Fig. XII.) A small collection of lymphocytes and a few plasma cells lead to no diagnostic inference, for they are common in chronic mastitis (see Fig. XIII), and even occur in the area of the breast around a fibro-adenoma. (See Fig. XIV from Fig. III.)

4, In some cases care must be taken not to miss cancer by mistaking epithelial cells of cancer which have been so altered by inflammatory and toxic changes that they resemble connective tissue, or inflammatory cells. The only method of determining the precise nature of such a growth is to cut a whole section of the tumour, hoping that in one or two outlying parts the true structure may have escaped the disguising change. However, this precaution is more necessary in uterine, intestinal, buccal, lingual, labial, and skin cancers than in breast cancer where ulceration and sepsis is less early.

5. I have 2 cases in which an unusual change occurred in the staining properties and size of the fibrous tissue cells removed from these suspicious areas in breasts. One Dr. Emery recently showed me, and I was able to show him the exact change I had noticed in a case I operated on six years ago. The pictorial reproductions of the type of affairs are not clear enough to show in this article, but it requires a good deal of careful examination to decide that it is a fibrous tissue change which causes this rare appearance.

6. Lastly, there is the early condition of cancer itself. Its microscopical appearance fortunately, as a general rule, is unmistakable, the epithelial cells invade the breast and the fat in its immediate vicinity, although their invasion is not advanced enough to produce retraction of the skin or to flatten the normal curve.

I have condensed an instructive little paper kindly given me by Dr. Whitfield, upon the method he usually employs in the diagnosis of Paget's disease of the nipple in its early stages.

The disease always begins either on the nipple itself or on the areola, and is unilateral. In the earliest stage of all there may be only a red and scaling surface with very little moisture present, but even in this stage careful examination reveals a card-like infiltration, which is absent in the simple eczematous eruptions. In syphilitic eruptions the induration is much thicker, giving to the finger the sensation of a small lump. The prominence of the nipple is lost extremely early in the disease, and it has already disappeared by the time that the patch is as large as a florin. The following microscopic examination is always sufficient to establish the diagnosis even in an an early case. Some scales from the dry stage or scrapings obtained with the edge of a microscopic slide in the erosive stage are mounted in 40 per cent. potash solution, and examined immediately with $\frac{1}{6}$ in. objective. There will be seen embedded in the scale or floating in the serous discharge bodies with a well-defined double outline, usually showing two concentric rings and a central nucleus (see Fig. XV). The bodies are slightly smaller than the ordinary squamous epithelial cell, and they vary in size.

Finally, the necessity for the early treatment of cancer is well known by all. In my earliest surgical days it was practised by Lord Lister, Mr. Godlee, Mr. Watson Cheyne, and latterly it has been advocated by Mr. Butlin and Mr. Mayo Robson. With a view to that end I humbly venture to point out the necessity for an earlier recognition of cancer when it affects the breast.

The City of London Lying-in Hospital is one of our oldest lying-in institutions. It was founded in 1750, and during the last century and a half has done excellent work. By the terms of its foundation it can only assist married women, and in addition to the indoor, it has an outpatient department, where patients are attended at their own homes by a midwife, who, if necessity arises, calls in one of the district surgeons of the institution. Any poor married woman is eligible as an in-patient, but out-patients are limited to those residing within a certain distance of the hospital. During the last year the in-patient and outpatients together amounted to 3,211 , the highest number yet reached, with only 3 deaths. The institution is also a school for midwives and monthly nurses, and during the past year 48 of the former and 107 of the latter were duly trained. Founded on its present site, it has stood the wear and tear of many generations; but, owing to increase of sanitary knowledge and the necessity of adapting it to present needs, it has become necessary practically to rebuild the hospital. The governors are to be congratulated on the success they have achieved in carrying on the work for some time past on temporary premises. It is hoped that by the end of the year the hospitai may once more be established in its old quarters, with all the improvements of modern science, and able to continue in the future the work so successfully accomplished in the past. The institution can be confidently recommended to the support of the charitable. The rebuilding has necessarily involved great expense, and practical benevolence cannot be better directed than in supporting so useful a work. 